\title{
Le clip musical et l'interaction texto-visuelle par écran
}

\section{Music video and texto-visual screen-based interaction}

\author{
Natalia Pimonova
}

Université Sorbonne Paris Nord-Paris 13, ED Erasme (Pléiade), France

\begin{abstract}
Résumé. Cet article porte sur la dimension visuelle de la chanson populaire, c'est-à-dire sur le clip musical, considéré comme l'ensemble synthétique de performances visuelle, textuelle et sociale. Les approches théoriques sont l'analyse du discours, l'analyse interactionnelle et les popular music studies. La problématique de la recherche est une marginalisation des clips musicaux sur le plan de la recherche en sciences humaines et sociales. Dans l'intention de répondre à cette problématique, nous visons à montrer comment les deux sens distaux, la vue et l'audition, contribuent aux interactions effectuées par écran et comment la perception audiovisuelle de la musique est formée. Les sensorialités écraniques sont mobilisées par les textes et les clips musicaux pour provoquer les émotions du public et transmettre le message principal d'un titre musical. L'étude porte sur l'analyse des clips vidéo des chansons sélectionnées et la distinction des catégories des moyens utilisés pour interagir avec le public et pour influencer les sens humains tels que : émoticônes, signes et couleurs, langage corporel, emprunts et nouvelles unités lexicales. Le corpus est constitué des chansons officiellement certifiées pour leurs ventes importantes et listées par le site du SNEP (Syndicat National de l'Edition Phonographique).
\end{abstract}

\begin{abstract}
This article examines texto-visual dimension of a mainstream song, i.e. music video that is considered as a combination of visual, textual and social (interactional) performances. Main theoretical approaches are discourse analysis, interaction analysis and popular music studies. Human and social sciences often exclude music videos from their research data. The purpose of this study is to show how two distant senses, vision and hearing, contribute to screen-based interactions and how audiovisual perception of music is formed. Screen-based sensorialities in music lyrics and videos serve to bring out emotions of the audience and to express the main message of a song. So, the article considers music videos as a research data and presents a classification of phenomena that help to influence human senses: emoji, signs and colors, body language, loanwords and new lexemes. The research data consists of songs that were officially certified for their high sales by The National Syndicate of Phonographic Publishing (SNEP).
\end{abstract}




\section{Introduction}

Le développement des technologies a joué un rôle important dans la perception de la musique. La chanson est devenue une forme multimédiatique qui se regarde autant qu'elle s'écoute via les clips musicaux, principalement diffusés sur Internet. En raison de la dématérialisation de la musique, l'évaluation des ventes et du succès d'un titre musical est calculée par les ventes digitales et non physiques. C'est pourquoi le format Internet des clips musicaux est privilégié dans cet article. L'objet de ce travail est la dimension visuelle de la chanson, sans oublier sa dimension textuelle. En premier lieu, le clip musical, considéré comme l'ensemble synthétique de performances visuelle, textuelle et sociale. En second lieu, le texte musical (ou les paroles de la chanson) qui rejoint les éléments lexicaux et sociaux.

Les sens proximaux sont inclus dans la perception des informations par écrans (smartphones, Apple Touch, expérience 4D). Néanmoins, l'objectif de l'article présent est de montrer comment les deux sens distaux, la vue et l'audition, contribuent aux interactions effectuées par écran. Cette recherche comprend une analyse des sensorialités écraniques, elles sont mobilisées par les textes et les clips musicaux pour provoquer les émotions du public et transmettre le message principal d'un titre musical. Un des moyens de le transmettre est à travers les sens décrits ou présents dans la chanson (notamment l'audition et la vue). Les interactions par écran comprennent la perception multifacette, c'est-àdire, elles offrent au public la possibilité de percevoir le message de la chanson en écoutant ses paroles et en regardant le clip vidéo en même temps.

La problématique principale est la marginalisation des clips musicaux sur le plan de la recherche en sciences humaines et sociales. Pourtant, la chanson commerciale occupe la place centrale sur le plan socio-économique (elle est au cœur de l'industrie musicale française). La présente recherche vise à montrer la façon dont le clip musical contribue au sens de la chanson ainsi qu'à analyser la formation de la perception audiovisuelle lors du visionnage d'un vidéo-clip.

La question de recherche est celle de la construction de la représentation de la société française contemporaine dans les clips vidéo et les textes des chansons commerciales. La dimension textuelle de cette recherche est introduite en analyse de l'interaction et en analyse du discours. Les domaines de la sociologie de culture et des popular music studies complètent l'analyse effectuée. Pour sa part, l'analyse visuelle a déjà été effectuée dans les articles suivants : Lebrun B. 2012 ; Gaudin A. 2018 ; Straw W. 2018 ; Rossi J. 2018 ; Boidy M. 2018. Cette étude contribue donc à l'analyse du clip vidéo et propose l'analyse du discours musical afin d'étudier de manière aboutie la corrélation entre les dimension visuelle et textuelle de la chanson. Ces deux dimensions comprennent plusieurs éléments et créent l'image complète d'une chanson, afin de la transmettre au public telle quelle.

Cet article se divise comme suit : l'introduction indique l'objectif et la problématique de la recherche; la première partie explique le cadre théorique, la méthodologie et le choix du corpus ; ensuite, la deuxième partie se consacre à l'analyse des phénomènes visuels et interactionnels; puis, la troisième partie présente les travaux réalisés sur des phénomènes lexicaux; enfin, la conclusion apporte un regard critique sur les résultats et introduit quelques perspectives de cette recherche.

\section{Cadre théorique et méthodologique}

La dimension visuelle détient actuellement une place prépondérante dans l'industrie musicale. D'un côté, le clip musical porte un caractère promotionnel, il est transmis à la télé et est accessible sur Internet, YouTube et d'autres plateformes digitales basées sur les informations en forme de vidéo. Généralement, « le vidéo-clip constitue un objet central dans le vaste univers audiovisuel contemporain " (Gaudin, 2018 : 97). De l'autre côté, le rôle des textes musicaux et de la musique elle-même ne peut pas être diminué - ces deux éléments constituent la base de la chanson. Will Straw affirme que « au-delà de YouTube, le clip vidéo joue désormais un rôle assez secondaire dans les scénarios possibles concernant le futur (et la renaissance) de l'industrie musicale » (Straw, $2018: 187$ ).

La présente recherche s'est surtout concentrée sur des sensorialités écraniques, nous proposons donc d'analyser les chansons sélectionnées d'un point de vue visuel (l'utilisation des émoticônes, des signes, des couleurs et du langage corporel dans les clips musicaux) ainsi que textuel (des emprunts et des nouvelles unités lexicales présents dans les paroles des chansons). Les émoticônes, originaires des réseaux sociaux numériques, contribuent à l'hybridité de la dimension texto-visuelle de la musique. Les signes et les couleurs produisent l'effet esthétique et portent le caractère émotionnel. Le langage corporel englobe les mimiques, la gestuelle et la danse utilisés dans les clips vidéo. Les emprunts et les nouvelles unités lexicales contribuent à la formation du discours musical étant le discours plurilingue et fortement métissé. Ces catégories lexico-visuelles permettent d'interagir avec le public, d'influencer les sens humains et de traiter les phénomènes sociaux, tels que le stéréotype social sur l'utilisation excessive des outils électroniques, l'antiracisme et le féminisme.

Dans ce contexte, l'approche méthodologique choisie est qualitative. Le but est de faire l'analyse multimodale de l'interaction artiste-public, des clips vidéo et du rapport image-texte, et enfin de montrer comment la parole n'existe pas sans le corps et comment la chanson influence les perceptions sensorielles par écran. Les approches théoriques sont l'analyse du discours, l'analyse interactionnelle et les popular music studies. Christine Develotte et Marie-Anne Paveau décrivent la multimodalité de la chanson telle qu'elle est présentée sur Internet : « ...il s'agit d'espaces multimodaux faisant co-exister des éléments graphiques, iconiques et picturaux, statiques ou mobiles, et des fonctions de communication synchrones et asynchrones... » (Develotte et Paveau, 2017 : 205). Dans un même ordre d'idées, Nicolas 
Auray insiste sur «l'intégration des écritures et des images » et parle d'une « nouvelle économie des signes sur l'espace de l'écran [...] caractérisée par l'intégration de systèmes expressifs appartenant à plusieurs formats, image, texte et son, dans le même média » (Auray, $2004: 97$ ).

\subsection{Choix du corpus et sa classification}

Des clips musicaux d'artistes francophones et anglophones en leur version Internet sont étudiés dans cet article. Afin de constituer le corpus de travail, les outils numériques suivants sont utilisés : le site des certifications musicales - SNEP (Syndicat National de l'Edition Phonographique) pour sélectionner des titres musicaux ayant les meilleures ventes (cela constitue la garantie que ces chansons sont au sommet des productions musicales contemporaines); la plateforme digitale YouTube pour collecter les clips musicaux des chansons sélectionnées; les sites Paroles2chansons et Genius pour trouver la version texte des chansons analysées.

Nous avons choisi des chansons récentes $(2013$ - 2018) pour faire l'analyse de l'image et évaluer sa contribution au sens de la chanson. La liste des chansons choisies pour l'analyse figure dans l'annexe (Voir Annexe 1).

Tous les titres musicaux sont divisés en groupes selon les moyens d'expression les plus utilisés dans chaque titre. Chaque catégorie représente un phénomène visuel ou lexical qui contribue le plus à la formation du message de la chanson. Les chansons ont été classées selon les caractéristiques suivantes :

- Émoticônes (Katy Perry « Roar » et Soprano « Mon précieux »);

- Signes et couleurs (The Carters « Apeshit » et Black M « Death Note »);

- Langage corporel (Childish Gambino « This is America » et Chilla « Si j'étais un homme ») ;

- Emprunts et nouvelles unités lexicales (Aya Nakamura « Djadja » et Soprano « Mon précieux »).

\section{Phénomènes visuels et interactionnels}

Selon ce qui précède, la transmission de l'idée complète de la chanson et l'interaction artiste-public sont possibles grâce à la coexistence de la parole et du corps. À cet effet, nous proposons d'inclure le terme discours dans l'analyse de la dimension visuelle de la chanson. L'approche multimodale implique que le discours comprend plusieurs phénomènes et les termes discours et interaction sont mutuellement dépendants et connectés. Catherine Kerbrat-Orecchioni explique que le terme de « discours en interaction » remplace aujourd'hui celui d' « interactions » (Kerbrat-Orecchioni, 2006 : 138). Le croisement des moyens d'expression visuels et lexicaux conditionne l'existence de pratique visio-discursive. «L'interaction s'organise donc non seulement sur la base, fondamentale, de l'organisation verbale des tours de parole, mais aussi à travers l'exploitation située des ressources multimodales, à travers les gestes, la disposition des corps dans l'espace ainsi que le recours à des artefacts variés » (Kerbrat-Orecchioni, 2005 : 131 - 132).

\subsection{Emoticônes}

Une grande partie de la communication est virtuelle, elle se passe à travers des smartphones et des réseaux sociaux. Cette interaction via Internet a développé un système des émoticônes souvent utilisés dans les messages ou les publications sur des réseaux sociaux. L'utilisation des émoticônes dans des clips musicaux permet de mettre l'accent sur le problème d'utilisation excessive des téléphones portables. Nous présentons donc deux titres musicaux qui parlent de l'influence des technologies sur notre vie quotidienne : « Roar » par Katy Perry (produit aux États-Unis) et « Mon précieux » par Soprano (produit en France).

Il existe de nombreuses formes d'intégration des émoticônes dans le clip musical. Par exemple, le clip musical de Katy Perry est devenu le premier clip vidéo (en version lyrics vidéo) où les paroles de la chanson sont présentées en forme de messagerie et de communication à l'aide des émoticônes. Au début de la vidéo $(0 \mathrm{~min} 08-0 \mathrm{~min} 10)$, on voit l'artiste prendre un téléphone portable dans ses mains dès qu'elle se réveille. Puis, on voit la discussion menée entre les amis où la plupart des mots sont remplacés par les émoticônes. Par exemple : l'image de la langue à la place du mot langue ou l'émoticône avec un singe qui couvre sa bouche par ses pattes utilisé à la place de dire " silencieusement » ou « en silence » (voir Figure 1): 


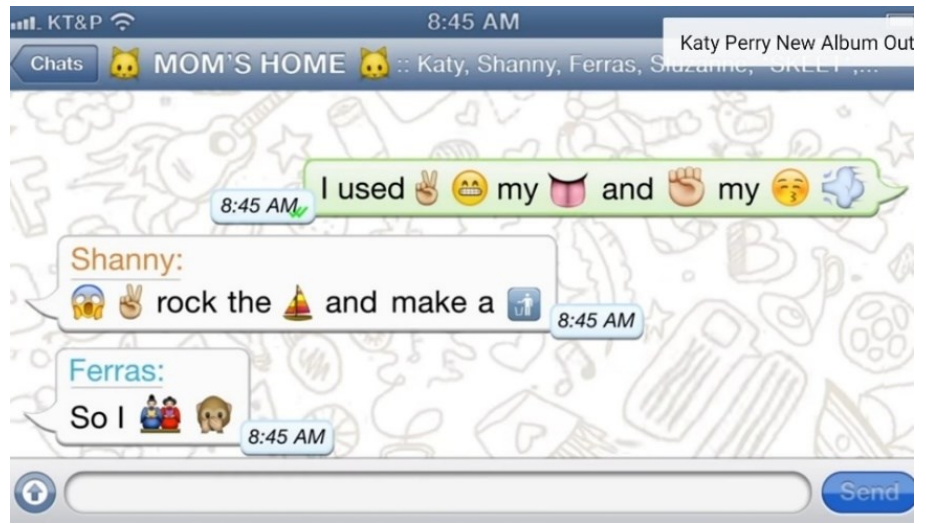

Figure 1. La capture d'écran du clip vidéo « Roar » par Katy Perry.

Les sens inclus dans la perception de cette vidéo sont :

- L'audition : on entend la sonnerie du téléphone portable au début de la vidéo ;

- La vue : dans le clip on voit l'application du messenger et la communication à l'aide des émoticônes. Cette vidéo représente un texte technographique (car il y a la co-présence de texte et d'image).

En ce qui concerne le clip musical « Mon précieux » par Soprano, le thème de la surutilisation des téléphones portables devient central. Les sens suivants sont inclus dans la perception de cette vidéo :

- Le toucher : exprimé verbalement, " je te prends par la main » ou " je te caresse le visage » (extrait de Soprano «Mon précieux », lignes 2 et 3 ). Alors, on constate un contact physique entre un être humain et son smartphone.

- La vue : exprimé verbalement, «mes yeux sont dans les tiens » (extrait de Soprano « Mon précieux », ligne 6) et visuellement, il y a la présence de l'écran dans le clip, Soprano et sa famille qui sont enchantés par un téléphone portable et regardent sans arrêt dans son écran. P.S. : la présence de l'écran dans l'écran forme un phénomène de double image.

Le clip se présente sous la forme d'une métaphore sur un téléphone portable qui devient un membre de la famille de l'artiste, son meilleur ami et confident. Les émoticônes, les likes, les icônes des applications les plus utilisées et les images pixelisées sont présents tout au long du clip (voir Figure 2). Le personnage principal ne se sépare jamais avec son smartphone, il reste enfermé dans la vie virtuelle, dans la non-réalité. Bien que le chanteur mette en avant le problème de la dépendance aux appareils électroniques, on peut remarquer la publicité du produit Wiko (une marque commerciale du fabricant des téléphones) au sein de son clip musical.

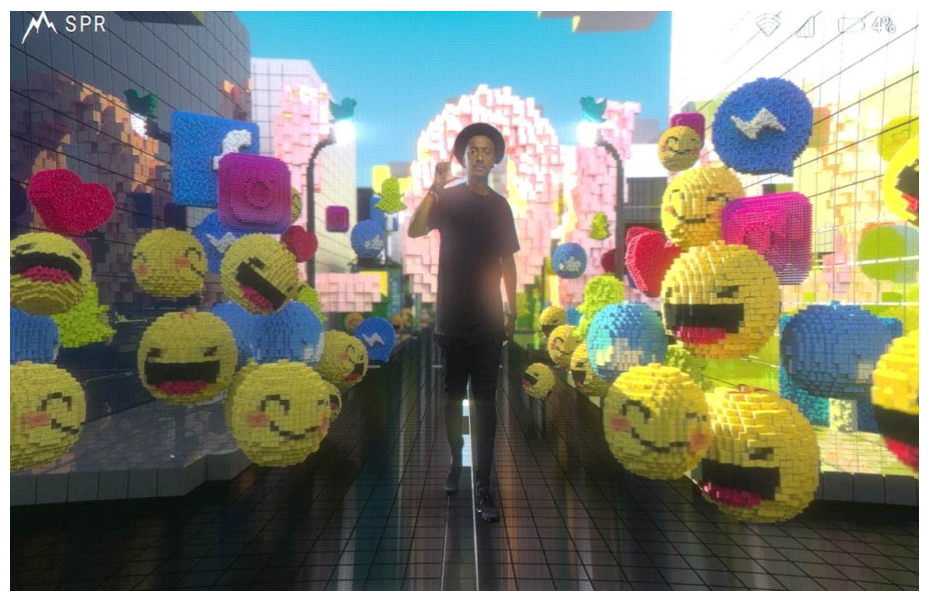

Figure 2. La capture d'écran du clip vidéo « Mon précieux » par Soprano.

Au niveau lexical, le titre musical de Soprano est riche en phénomènes linguistiques et sociaux qui sont traités dans la troisième partie de l'article. 


\subsection{Signes et couleurs}

La symbolisation et la coloration des clips musicaux sont les deux moyens d'expression forts et souvent utilisés dans l'industrie musicale. Afin d'illustrer ces aspects visuels, nous avons sélectionné les clips suivants : « Apeshit» par The Carters (produit aux États-Unis) et « Death Note » par Black M (produit en France). Le clip musical " Apeshit » s'est plutôt concentré sur des signes et des symboles implicites. Alors que le clip «Death Note » met l'accent sur des couleurs utilisées.

Dans cette catégorie, les deux sens distaux sont distingués :

- La vision : exprimé au niveau de la symbolisation du clip et la présence des objets ou des tableaux qui ont la signification spécifique ;

- L'audition : exprimé musicalement dans le titre "Apeshit» où on entend des cris des singes. Cette référence sur la jungle, le zoo et la sauvagerie est également reflétée par le titre de la chanson - le mot apeshit (en anglais « des excréments des singes ») est utilisé dans l'expression to go apeshit, elle signifie « se déchaîner ".

La symbolisation de la chanson «Apeshit est majoritairement illustrée par des peintures et des monuments utilisés dans le clip vidéo. Guillaume Kientz, conservateur du Louvre au département des peintures, estime qu' " on a tendance à opposer la culture des musées, élitiste, à celle des gens qui écoutent Beyonce. Pour une fois, nous ne sommes pas dans un rapport de confrontation mais dans une réciprocité, une alchimie qui fonctionne très bien » (Constantin, 2018: 34). Le clip musical «Apeshit » cherche à installer le rapport entre la culture hip hop (considérée marginale) et la culture classique (considérée élitiste). Le couple The Carters ${ }^{1}$ sert à découvrir et à établir le lien entre ces cultures, les artistes rendent l'hommage à la culture pop et hip-hop, à la culture et au physique des personnes noires.

$\mathrm{Au}$ niveau textuel, il est important de noter l'utilisation du lexique argotique. Ce lexique est habituel pour le genre musical de hip-hop, mais non-habituel pour la culture dite «classique ». Sous prétexte du statut élevé de l'industrie musicale, nous pouvons faire l'hypothèse que Jay $\mathrm{Z}$ et Beyonce essaient de normaliser le lexique populaire et argotique, considéré comme « le lexique hors norme ».

Au niveau visuel, on peut remarquer un grand nombre de significations implicites. Le sens des peintures exposées au Louvre et utilisées dans le clip est expliqué par des professionnels du Louvre (Bindé, 2018 : page web). Par exemple :

- «La Joconde » est une peinture la plus connue au monde, elle est « une star mondiale » comme les artistes The Carters (voir Figure 3);

- «La Victoire de Samothrace » (le monument de la déesse Niké) est un signe de la réussite sociale et du triomphe des femmes noires;

- «Le Radeau de la Méduse » est un symbole de l'esclavage ;

- «Le Sacre de Napoléon » représente une cérémonie du couronnement de l'impératrice Joséphine dans la cathédrale Notre-Dame de Paris. C'est un symbole du couronnement de Beyonce (un tribut à la force des femmes et au féminisme) et de Jay Z.

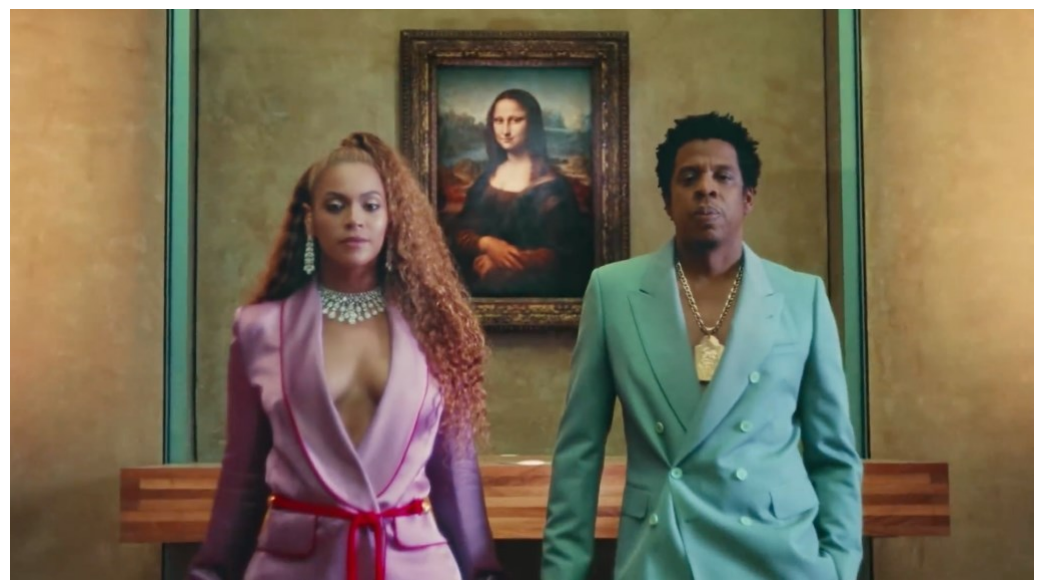

Figure 3. La capture d'écran du clip vidéo « Apeshit » par The Carters.

$\mathrm{Au}$ niveau texto-visuel, Jay $\mathrm{Z}$ et Beyonce manifestent leurs puissance et richesse. En premier lieu, le clip est tourné au Louvre (l'ancienne résidence royale, un des musées les plus connus au monde). En second lieu, The Carters

\footnotetext{
${ }^{1}$ The Carters - est un ensemble de deux musiciens (Beyonce et Jay Z) formé d'un couple marié. Dans la chanson « Apeshit» ils se comparent aux époux ayant le statut royal, l'empereur Napoléon Ier et Joséphine de Beauharnais.
} 
construisent l'image « royale » verbalement, ils comparent leur musique et leur statut social avec le statut des familles royales et présidentielles :

Texte original

"...I'm like Chief Keef meet Rafiki, who been Lion King to you

[...]

Motor cade when we came through

Presidential with the planes too... »

(extrait de The Carters « Apeshit », lignes 49, 52-53)

La coloration des clips vidéo peut devenir un moyen d'expression significatif et contribuer à la construction du sens de la chanson. Nous proposons d'analyser les couleurs selon leur signification dans la culture occidentale. Par exemple, le clip musical de la chanson « Death Note " par Black M est réalisé en noir et blanc. Le thème de la mort est exprimé par la couleur noire, selon la mentalité de la société occidentale moderne. Dans cette vidéo, la couleur rouge est également présente, sa signification peut être assez ambiguë : d'un côté, la couleur rouge peut signifier la passion, la chaleur et la vitalité, la vie elle-même et l'énergie ; d'un autre côté, on l'associe au sang, à l'enfer et à la luxure. Alors, la signification de cette couleur dans « Death Note » est contradictoire, cela peut définir l'opposition vie-mort ou le support de la couleur noire pour exprimer la douleur et la souffrance.

La capture d'écran illustre le moment de la chanson où le rappeur Black M a le cahier Death Note dans ses mains (voir Figure 4). Ce cadre attire l'attention du public au cahier et à sa signification (la capture d'écran est prise sur 3min57).

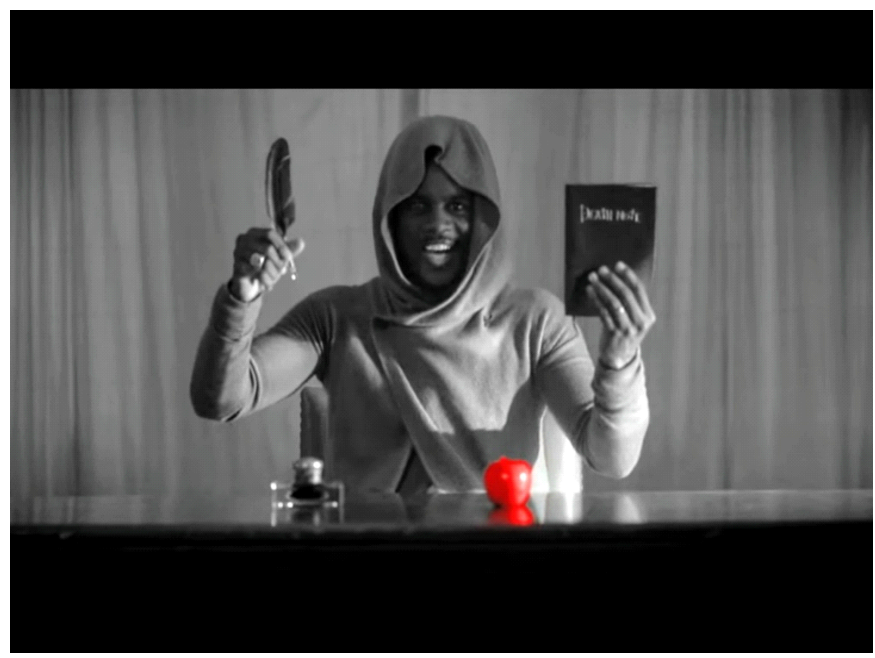

Figure 4. La capture d'écran du clip vidéo « Death Note» par Black M.

Parallèlement à la coloration du clip vidéo « Death Note », d'autres moyens d'expression deviennent les symboles de la mort : le titre de la chanson « Death Note » signifiant une " lettre d'adieu »; le rappeur portant un capuchon (une métaphore visuelle sur une figure de la mort vêtue d'un grand manteau noir à capuche); la pomme rouge, une interprétation possible d'une pomme empoisonnée (un fruit magique utilisé pour tuer un humain).

\subsection{Langage corporel}

Ainsi que les effets visuels et le texte des chansons, le langage corporel contribue à l'expression du sens de la chanson et des émotions des artistes. Les mimiques et la gestuelle sont des outils de l'interaction efficaces et répandus, ils servent à construire la communication artiste-public diversifiée. Lorenza Mondada soutient que " ...l'interaction s'organise grâce à une pluralité de ressources multimodales : les gestes, les regards, les postures corporelles, les mouvements, l'agencement spatial des participants » (Mondada, $2005: 111$ ).

Un des sens distaux, la vue, permet de percevoir les danses, les mouvements et les mimiques d'un ou d'une artiste. Les deux chansons, «This is America » par Childish Gambino (produit aux États-Unis) et «Si j'étais un homme » par Chilla (produit en France), sont présentées dans cette catégorie.

La chanson de Childish Gambino «This is America » est consacrée aux violences et injustices réalisées derrière la culture populaire et les médias (notamment les violences de la police contre les personnes noires aux Etats-Unis).

Le sens de la chanson est exprimé au niveau visuel par : 
- La danse et les expressions faciales du chanteur et des danseur.euse.s - elles sont prononcées, et même excessives, nous faisons l'hypothèse qu'elles cherchent à montrer la perception raciste ${ }^{2}$ des personnes noires (voir Figure 5);

- La posture du chanteur avec un pistolet - cette position présente une référence culturelle connue aux ÉtatsUnis. L'artiste joue le rôle de Jim $\mathrm{Crow}^{3}$, qui est devenu le symbole du racisme et, ultérieurement, de l'antiracisme (voir Figure 6);

- La présence des agents de police chassant l'artiste (une personne noire) et des gestes imitant un pistolet dans les mains - ces moyens visuels expriment la violence policière régulière qui existe dans la société étasunienne.
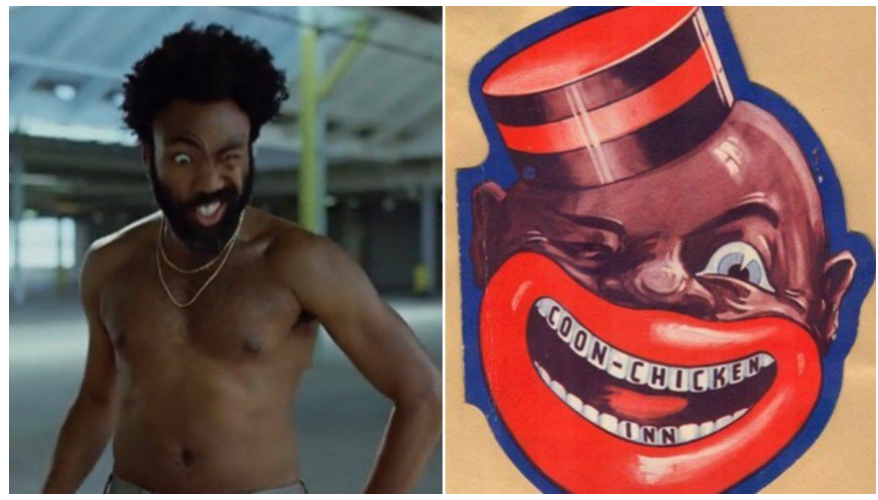

Figure 5. La capture d'écran du clip vidéo « This is America » par Childish Gambino.
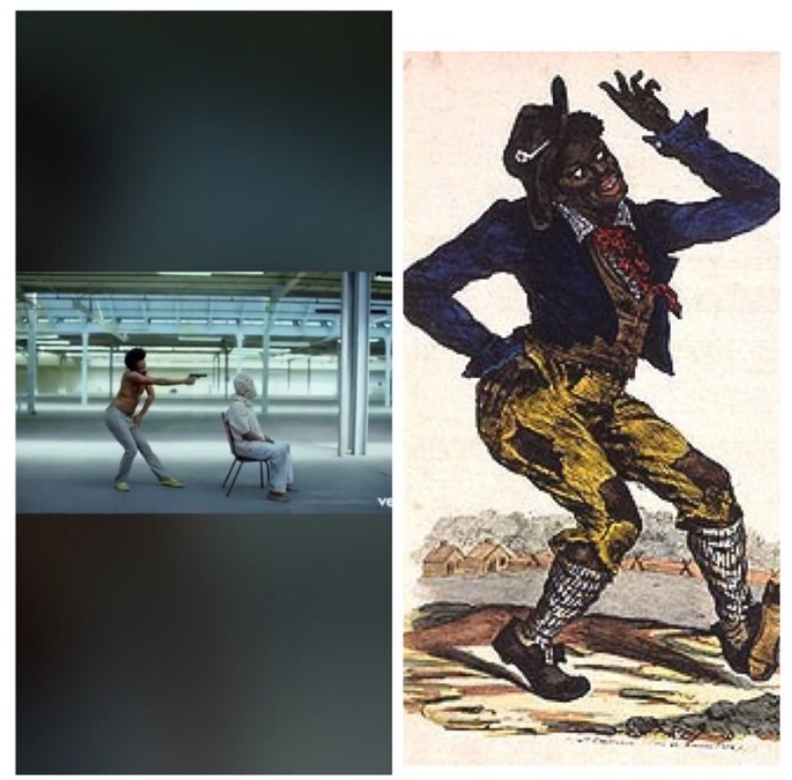

Figure 6. La capture d'écran du clip vidéo « This is America » par Childish Gambino.

La deuxième chanson de cette catégorie transmet son message douloureux à travers la danse et les mimiques d'une danseuse. La rappeuse Chilla dans son titre « Si j'étais un homme » parle du changement des rôles entre un homme et

\footnotetext{
${ }^{2}$ Au XIXème-XX siècles aux Etats-Unis, les capacités physico-artistiques et les expressions faciales excessives des personnes noires étaient ridiculisées et caricaturées. C'est un des traits caractéristiques de la perception et de l'image racistes.

${ }^{3} \mathrm{Jim}$ Crow - est un personnage incarnant un esclave d'origine africaine et popularisé par une chanson "Jump Jim Crow" (1828), pendant des décennies, Jim Crow était le symbole de la ségrégation et du racisme anti-noir. À présent, il apparaît comme le symbole du mouvement antiraciste.
} 
une femme. Au niveau lexical, l'artiste propose des sujets stéréotypés où un homme domine ou oppresse une femme. Par exemple :

\section{" J'te parlerai comme jamais tu ne parles à ta mère \\ Tu me seras redevable si je te paie un verre \\ J't'harcèle avec dix potes juste pour avoir ton numéro}

Il faudra que tu sois gentille si je t'emmène au restau' » (extrait de Chilla « Si j'étais un homme », lignes 1 - 4).

Au niveau visuel, on voit la danse d'un couple, le danseur et la danseuse changent des positions (soit lui, soit elle est derrière son partenaire) afin de représenter le changement de leurs rôles sociaux. Les souffrances de la femme sont montrées à travers des mouvements de son corps, les mimiques de la danseuse expriment la douleur, le désespoir et l'inquiétude (voir Figure 7).

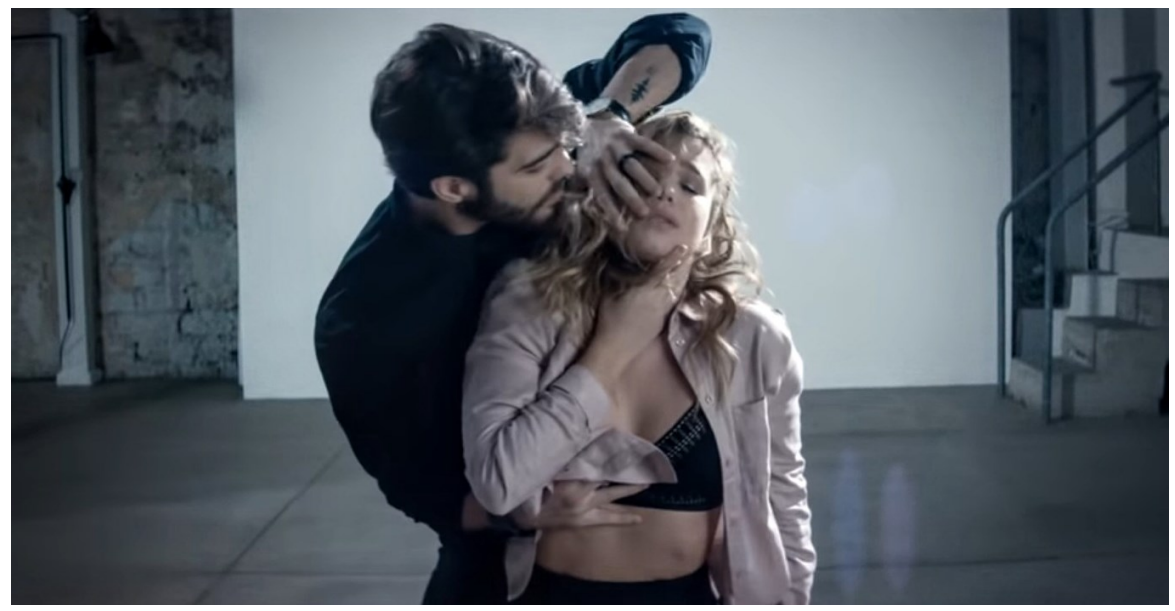

Figure 7. La capture d'écran du clip vidéo « Si j'étais un homme » par Chilla.

\section{Phénomènes lexicaux : emprunts et nouvelles unités lexicales}

Bien que la présente étude soit consacrée à la dimension visuelle de la chanson française commerciale contemporaine, nous voudrions faire quelques commentaires sur les textes analysés. Cette dernière catégorie observée dans notre recherche, porte sur des phénomènes lexicaux et leur rôle dans la création du sens des chansons.

Par exemple, la chanson d'Aya Nakamura « Djadja » est devenue un phénomène social et langagier grâce à son lexique. Le texte de ce titre musical est riche en phénomènes littéraires, poétiques et lexicaux à analyser. Par exemple, il contient du lexique étranger naturalisé et des emprunts de langues différentes (par exemple : que pasa espagnol, dead anglais et tchouffer nouchi, argot ivoirien) et des mots comme «djadja» (un menteur) ou " en catchana » (une position sexuelle). Le lexique familier et argotique sont principalement utilisés dans ce titre musical, en même temps, le texte ne perd pas sa poéticité grâce à la présence des métaphores, des répétitions, des rimes et du rythme. Les caractéristiques poétiques se trouvent dans toutes les dimensions de la chanson commerciale : textuelle, visuelle et sonore. Pour sa part, Gilles Bonnet « envisage la rime comme caractéristique prégnante pour définir le genre " chanson », du fait de ses qualités esthétiques (recherche lexicale, formules fantaisistes), mais aussi de son interpénétration avec la voix et l'accompagnement» (Bonnet, $2013: 176)$.

\section{«... Hello papi mais qué pasa \\ [...]}

Oh Djadja (oh Djadja)

Y a pas moyen Djadja (y a pas moyen Djadja)

J'suis pas ta catin Djadja, genre en Catchana baby tu dead ça

[...]

Tu jouais un rôle, tu finiras aux enfers... » (extrait d'Aya Nakamura « Djadja », lignes 2, 10 - 12, 21).

La deuxième chanson analysée dans ce chapitre est « Mon précieux » par Soprano, elle comporte plusieurs phénomènes langagiers, y compris le lexique des technologies et des innovations :

«Mes yeux dans tes applis, baby

$[\ldots]$ 
Tu es ma secrétaire, tu gères mon organisation

Tu allèges mes neurones grâce à tes notifications

Plus besoin d'aller voir la famille, vu que tu me les follow

Pour leur prouver que j'les aime, je n'ai qu'à liker leurs photos » (extrait de Soprano " Mon précieux », lignes $12,21-24)$.

Ce lexique est souvent utilisé dans le texte de cette chanson, mais il est déjà naturalisé. Le développement des technologies et des innovations techniques explique la présence des phénomènes lexicaux décrivant les activités réalisées sur des réseaux sociaux numériques («tes applis », «ton regard pixélisé», « tu me les follow », «liker leurs photos », etc.).

En ce qui concerne le titre de la chanson « Mon précieux », il comporte également un phénomène lexical à observer - le lexique hypocoristique. Normalement, ce lexique sert à exprimer l'affection pour une personne qu'on aime. Cependant, c'est le portable qui est devenu l'objet d'amour, il joue le rôle d'une personne à qui on s'adresse avec les mots « mon précieux ». Suivant cette idée, il est intéressant de constater que le titre de la chanson a le caractère affectif et sarcastique en même temps. D'autres exemples du lexique affectif sont utilisés dans le texte de cette chanson : "puis je te caresse le visage pour voir si tout va bien », «tout le monde t'aime dans ma famille, baby » ou " je ne sais plus vivre sans toi à mes côtés » (extraits de Soprano « Mon précieux », lignes 3, 8, 12).

En résumé, le lexique étranger figure dans les chansons commerciales contemporaines, notamment les mots doux et les mots décrivant des activités ou des applications numériques qui sont empruntés de la langue anglaise. Afin de révéler le lien entre le lexique des chansons analysées et l'activité humaine sur le web, nous avons choisi deux exemples du discours musical apparaissant sur les réseaux sociaux, notamment sur Twitter et Instagram (voir Figures 8, 9). Les paroles de la chanson «Djadja » par Aya Nakamura sont mis dans le texte de la publication Twitter en forme de l'hashtag \#yapasmoyendjadja, dans le but d'exprimer ses doutes sur l'honnêteté d'un homme politique. Puis, le lexique présent dans la chanson " Mon précieux » par Soprano est également utilisé dans une publication web dans l'intention d'exprimer l'affection pour un objet (des biens matériels) et non pour une personne qu'on aime.

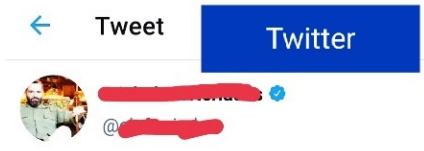

@EmmanuelMacron Monsieur Geagea, via ses colporteurs, insinue depuis l'annonce de votre geste généreux en faveur des étudiants Libanais en France qu'il est l'instigateur de cette décision. Or mon p'tit doigt me dit que ce n'est bas du tout le cas. \#YapasmoyenDjadja
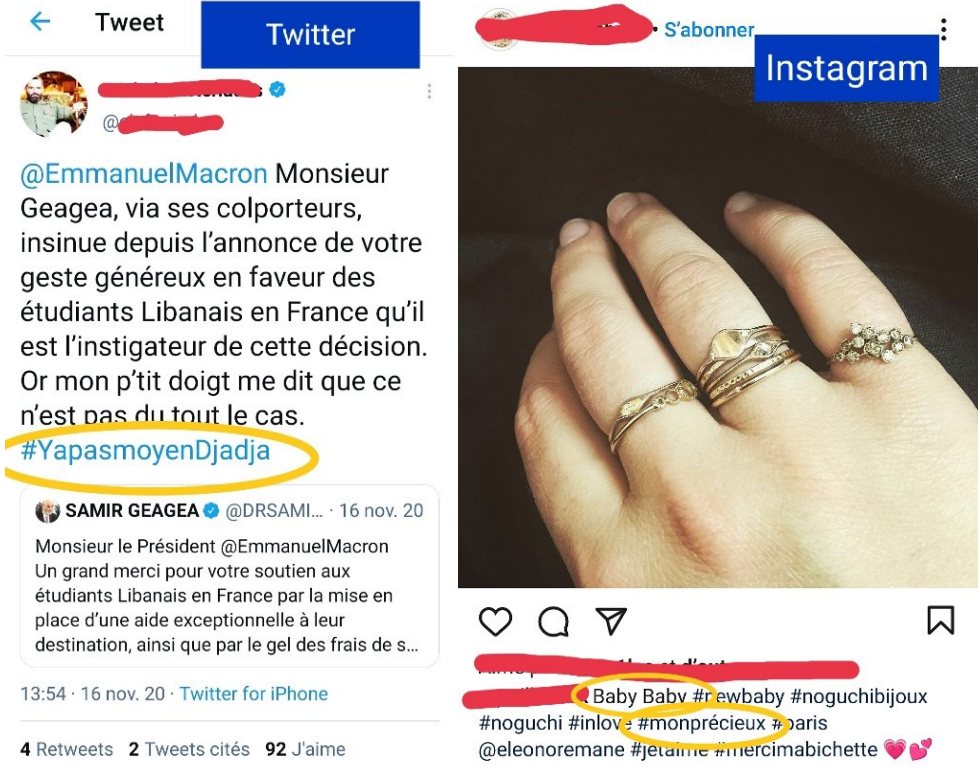

Figure 8. La capture d'écran d'une page Twitter.

Figure 9. La capture d'écran d'une page Instagram.

Selon ce qui précède, le discours musical, des emprunts et des phénomènes appartenant à la culture étrangère sont présents sur le web. Cela illustre la connexion existante entre les musiques actuelles hétérogènes et les réseaux sociaux qui nous accompagnent quotidiennement.

\section{Conclusion}

Pendant ces dernières décennies, la musique populaire existe dans la dimension visuelle, il est logique d'inclure des sensorialités écraniques dans l'étude de la chanson et de montrer l'investissement des sens distaux dans des interactions 
effectuées à travers le clip musical. Étant donné que la chanson est actuellement accessible sur Internet, il est possible de la traiter comme un document numérique. Ghitalla indique que :

« ...Le document numérique est tout d'abord hétérogène du point de vue des formats sensoriels exploités : la vue et l'ouïe essentiellement (voire la proprioception pour les effets de stéréophonie auditive, d'animation visuelle et de gestion de l'espace-écran). Plutôt que « multimédia », le document numérique apparaît comme polysensoriel... » (Ghitalla, 1999 : 100).

La centralité du clip musical reste une question contradictoire, est-ce que c'est le texte ou l'image qui prime ? Cela dépend de la sélection de corpus, certains titres musicaux se focalisent sur l'image et certains sur le texte (ou les deux).

D'une part, c'est la dimension textuelle, donc langagière, qui reste dominante dans la chanson populaire. Parmi tous les titres musicaux qui ont été analysés au sein de travail réalisé pour la thèse, il y avait certains où le sens est construit par des paroles de chanson, sans la contribution signifiante du clip vidéo. Si les éléments textuels dominent dans la chanson française commerciale, on peut parler de « la discursivisation de la musique » (Péquignot, 2018 : 122), c'est-à-dire, d'une perception de la chanson étant un texte relativement court, poétique et portant un message complet. Dans ce cas, le rôle de la musique et de l'image devient secondaire dans la construction du sens de la chanson.

De l'autre part, l'importance des textes des chansons a diminué pendant le siècle des nouvelles technologies et des plateformes digitales, et le centre d'attention est actuellement sur l'image et sur le clip vidéo. Selon Marie-Anne Paveau, " sur Internet, de nombreux éléments visuels témoignent en effet de cette domination de l'image sur le langage ". (Paveau, 2017 : 309). En même temps, selon les résultats d'analyse effectuée dans cet article, l'image prime ou joue un rôle équivalent au texte pour construire le sens de la chanson populaire. Les moyens d'expression visuels et sonores sont souvent utilisés pour interagir avec le public, et le clip musical devient un outil d'analyse autosuffisant. À travers des couleurs, des vêtements, des gestes ou des mimiques utilisés dans les vidéos, il est possible de construire un rapport image-texte. Nous concluons que la contribution de la dimension visuelle à la mise en valeur du texte de la chanson commerciale contemporaine est considérable.

Les résultats obtenus au fil de ce travail aident à résoudre la problématique de la recherche. Nous avons prouvé le rôle important des sensorialités écraniques au sein de l'interaction effectuée par écran, notamment à travers les représentations textuelles et audio-visuelles de la société française. En même temps, la construction de l'image de société n'est pas réussie. La question de la représentation de la société française contemporaine a une perspective plus globale, et il existe d'autres dimensions de la chanson commerciale contemporaine (sociale, culturelle, musicale, politique, textuelle, etc.) lesquelles on puisse étudier. À titre d'exemple : la poéticité de la chanson commerciale qui sert à produire des effets esthétiques sur ses destinataires ; les thématiques et les stéréotypes sociaux les plus présents dans les titres musicaux analysés ; la possibilité de la transmission des langues à travers la musique populaire ; la connexion entre la société et la chanson créée par des moyens visuels, musicaux et textuels.

\section{Références}

Auray Nicolas. (2004). Sosies et avatars dans les jeux : entre écriture et image, L'image Socie. L'original et son double, 1er colloque international ICONE-IMAGE, Sens, Musées de Sens, p. 95-108.

Bindé Joséhine. (2018). L’incroyable clip de Beyoncé et Jay Z décodé tableau par tableau, Beaux-Arts, https://www.beauxarts.com/videos/lincroyable-clip-de-beyonce-et-jay-z-decode-tableau-par-tableau/, consulté le $23 / 03 / 2021$

Boidy Maxime. (2018). Corps, gestes, interpellations. Iconographie politique du format clip, Volume !, 14 : 2 | 2018 , p. 175-186, https://journals.openedition.org/volume/5581, consulté le 15/04/2021.

Bonnet Gilles (dir.). (2013). La Chanson populittéraire. Texte, musique et performance, Paris, Kimé. Volume !, 12 : 1 | 2015, p. 175-176, https://journals.openedition.org/volume/4674, consulté le 15/04/2021.

Constantin Sarah. (2018). Les Carter sont-ils devenus des œuvres d'art ?, revue Grazia du 13/07/2018 p. 34.

Develotte Christine, Paveau Marie-Anne. (2017). Pratiques discursives et interactionnelles en contexte numérique. Questionnements linguistiques, Langage et société, $\mathrm{N}^{\circ} 160-161$, p. 199-215, https://www.cairn.info/revuelangage-et-societe-2017-2-page-199.htm, consulté le 25/10/2020.

Gaudin Antoine. (2018). Le clip comme forme d'expression musico-visuelle : pour une esthétique de la relation musique-images, Volume !, p. 97-110, https://journals.openedition.org/volume/5556, consulté le 29/10/2020.

Ghitalla Franck. (1999). NTIC et nouvelles formes d'écriture. Dans : Communication et langages, $\mathrm{n}^{\circ} 119$, 1er trimestre 1999. Dossier : Les nouvelles technologies de la communication. p. 91-105, http://www.persee.fr/doc/colan_0336-1500_1999_num_119_1_2913, consulté le 29/10/2020.

Kerbrat-Orecchioni Catherine. (2005). Le discours en interaction, Paris, Armand Colin, p. 368

Kerbrat-Orecchioni Catherine. (2006). Comptes rendus, Revue française de linguistique appliquée, 2006/2 (Vol. XI), p. 138-144, https://www.cairn.info/revue-francaise-de-linguistique-appliquee-2006-2-page-138.htm, consulté le $11 / 05 / 2021$. 
Lebrun Barbara. (2012). Chanson et performance : mise en scène du corps dans la chanson française et francophone, Paris, L'Harmattan, p. 222.

Mondada Lorenza. (2005). Chercheurs en interaction. Comment émergent les savoirs. Lausanne : Presses Polytechniques et Universitaires Romandes, p. 144.

Paveau Marie-Anne. (2017). L'analyse du discours numérique. Dictionnaire des formes et des pratiques. Hermann Editeurs, Paris, p. 398.

Péquignot Julien. (2018). Clip et discours : pragmatique de l'énonciation, Volume !, 14 : 2 | 2018, p. 111-124, https://journals.openedition.org/volume/5572, consulté le 28/10/2020.

Rossi Jérôme. (2018). "L'homme au bouquet de fleurs » de Maxime Le Forestier : le clip comme approfondissement de la chanson, Volume!, $14: 2$ | 2018, p. 125-136, https://journals.openedition.org/volume/5575 , consulté le $15 / 04 / 2021$.

Straw Will. (2018). Le clip vidéo et ses contextes : 30 ans plus tard, Volume !, 14 : 2 | 2018, p. 187-192, https://journals.openedition.org/volume/5589, consulté le 27/10/2020.

\section{Outils de travail}

Site des certifications musicales - SNEP (Syndicat National de l'Edition Phonographique) http://snepmusique.com/lescertifications/

YouTube https://www.youtube.com/watch

Paroles2chansons https://paroles2chansons.lemonde.fr/

Genius, Song Lyrics \& Knowledge https://genius.com/

\section{Corpus de recherche}

Aya Nakamura « Djadja » 2018 https://www.youtube.com/watch?v=iPGgnzc34tY (Clip officiel) 695 millions de vues Black M « Death Note » 2017 https://www.youtube.com/watch?v=JYqb4cdRFDg (Clip officiel) 5 millions de vues

Childish Gambino « This is America » 2018 https:/www.youtube.com/watch?v=VYOjWnS4cMY\&ab channel=ChildishGambinoVEVO (Official Video) 731 millions de vues

Chilla « Si j'étais un homme » 2017 https://www.youtube.com/watch?v=Kn-lbI7MESI (Clip Officiel) 25 millions de vues

Katy Perry « Roar » 2013 https://www.youtube.com/watch?v=e9SeJIgWRPk (Lyric Video) 89 millions de vues

Soprano « Mon précieux » 2016 https://www.youtube.com/watch?v=OVmfGb8XKSg\&ab_channel=SopranoOfficiel (Clip officiel) 141 millions de vues

The Carters « Apeshit» 2018 https://www.youtube.com/watch?v=kbMqWXnpXcA 227 millions de vues ${ }^{4}$

\footnotetext{
${ }^{4}$ Nombre de vues des vidéos YouTube est calculé le 04/11/2020
} 